\title{
Espacios de trabajo geométrico personal de profesores de matemáticas en formación
}

\section{Personal geometric workspaces of math teachers in training}

Jonathan Cervantes-Barraza
Universidad del Atlántico, Barranquilla, Colombia.
Universidad del Atlántico, Barranquilla, Colombia.
María José Contreras-Vásquez
Universidad del Atlántico, Barranquilla, Colombia
Vanessa Paola Martínez-Fontalvo
Universidad del Atlántico, Barranquilla, Colombia.

Palabras clave: Espacios de Trabajo Geométrico, Profesores en formación, Parábola, Geometría Sintética, Geometría Analítica, Estrategia Investigativa Clínica.

\begin{abstract}
Objective: To characterize the personal geometric workspaces of teachers in training in the context of problems that involve the articulation between synthetic and analytical geometry. Method: It was carried out hand in hand with the theory of paradigms and geometric workspaces developed by Alain Kuzniak and Houdement. The research was developed with a qualitative approach and based on a clinical research strategy; a priori and a posteriori analysis was used as the research methodology and a sample of four mathematics teachers in training from a public university in northern Colombia. Results: The teachers' personal mathematical workspaces show key elements in the articulation between Euclidean and analytic geometry identified through the arguments and reasoning used in solving geometry problems. Discussion and Conclusion: The teachers' workspaces were characterized by activating the figural genesis more frequently by ratifying the information through the visualization pole in circulation with the real and local space pole. The proposed problem promoted the use of strategies where synthetic geometry was approached effectively as an exploratory route for the definitions of analytic geometry.
\end{abstract}

Keywords: Geometric Workspaces, Teachers in training, Parabola, Analytic Geometry, Synthetic Geometry, Clinical Research Strategy.

\section{ARTÍCULO DE INVESTIGACIÓN CIENTÍFICA Copyright $\odot$ By Educación y Humanismo \\ Editor: Dhayana Fernández Matos Universidad Simón Bolívar \\ Correspondencia: Vanessa Martínez vpaolamartinez@mail.uniatlan tico.edu.co}

Recibido: 30-05-2020 Aceptado: 10-09-2020 En línea desde: 15-12-2020

Cómo citar este artículo (APA): Cervantes-Barraza, J., Berrío-Valbuena,J., Contreras-Vásquez, M., y Martínez-Fontalvo, V. (2021). Espacios de trabajo geométrico personal de profesores de matemáticas en formación. Educación y Humanismo, 23(40), 1-17. https://doi.org/10.17081/eduhum.23.40.4083 


\section{Introducción}

La geometría se ha distinguido por ser una de las ramas de la Matemática que en gran medida ha conformado objetos de estudio que consecuentemente parten de la aplicación de sus métodos de enseñanza-aprendizaje hasta su implementación (Cabello, 2006; Gamboa y Ballesteros, 2009; Fernández, 2018). Los objetivos de estudios denotados, marcan un contexto en los campos de la educación que ostentan paulatinamente la conformación de herramientas teóricas y prácticas con miras al mejoramiento de los niveles de comprensión, una vez estos hayan pasado por su etapa de desenvolvimiento. Es por ello que todo este conglomerado de acciones precisa características y correlaciones que subyacen en los campos de educación de la geometría, en el cual evidentemente surge el reconocimiento e importancia de la misma.

La importancia de la geometría deriva de su influencia en el desarrollo de pensamientos, acciones y demostraciones que están presente en todas las etapas educativas del ser humano, es decir, abarcan sus primeros años de escolaridad hasta sus años de vida universitaria, en el cual sus elementos se sitúan en las elaboraciones de escenarios idóneos que refuercen la capacidad cognitiva (creación de conocimientos) y posterior implementación (ejecución de saberes), partiendo de énfasis pragmáticos que surjan y culminen dentro de los campos de educación (Fernández, 2018).

Numerosas investigaciones hacen hincapié en implementar la geometría como eje para desarrollar métodos de enseñanza- aprendizaje (Gascón, 2002; Montoya, 2014; Henríquez y Montoya, 2015; Fernández, 2018), dado que posee una diversidad de conceptos, propiedades, métodos deductivos-inductivos que permiten elaborar y plantear diversos procesos de análisis, cuestionamientos y realizar construcciones de figuras, sólidos que conlleven a los estudiantes en la construcción de demostraciones formales las cuales son muy importantes en el proceso de formación (Hernández, 2012).

A lo largo de la educación obligatoria, la enseñanza de la geometría aborda dos grandes enfoques, la geometría sintética y la analítica. La primera, se refiere a la implementación de instrumentos como lápiz, regla y compás en un plano sin referencia alguna para solucionar problemas geométricos, en cambio, la segunda, tiene que ver con el estudio de los objetos matemáticos, donde predomina la necesidad de implementar definiciones, axiomas que permiten que las figuras geométricas puedan ser representadas mediante fórmulas en un sistema de referencia denominado plano cartesiano (Del Río, 2018).

Estudios relacionados con la enseñanza y aprendizaje de la geometría han identificado una desarticulación entre conceptos, propiedades, métodos para el análisis y construcción de objetos entre la geometría sintética y analítica (Gascón, 2002; Montoya, 2014; Henríquez y Montoya, 2015). La disyuntiva entre lo sintético y lo analítico puede fomentar considerablemente un desacierto en el docente de Matemática en formación inicial lo que puede implicar que este solucione problemas geométricos aislando los conocimientos, conceptos o considerándolos como dos enfoques diferentes de la geometría. 
En particular, se ha documentado que el docente no percibe que los dos enfoques pueden ser usados en un mismo objeto geométrico, razón por la cual se genera la ruptura entre las geometrías (Di Blasi y Rodríguez, 2016; Álvarez, 2014; Santaló, 1961). Asimismo, los estudiantes consideran aspectos procedimentales sin identificar conceptos matemáticos relacionados con la geometría sintética y analítica (Ancochea, 2011). De igual manera, Di Blasi y Rodríguez (2016) y Álvarez (2014) perciben una ruptura entre estas dos ramas de la geometría pues los estudiantes divisan de manera aislada los conocimientos entre sí, donde lo ideal sería aprender los conocimientos del objeto geométrico desde el mismo punto de vista sintético y analítico, para que no se presente una ruptura que, como consecuencia, le haga perder sentido de conocimiento y capacidad al estudiante para reconocer que son enfoques del mismo objeto.

Estudios sobre la enseñanza de la geometría evidencian dificultades, como lo manifiesta Itzcovich (2005), autor que reconoce que en las instituciones de educación al momento de desarrollar los contenidos geométricos en los diseños curriculares no se tiene en cuenta la importancia de esta, ya que solo se busca que el estudiante aprenda los teoremas, axiomas y conceptos relacionados con la geometría y no a desarrollar situaciones problemas que permitan la articulación entre la geometría sintética y la analítica. Por su parte, Ramírez (2013) afirma que el tema de las secciones cónicas resulta muy sugestivo, puesto que está relacionado con uno de los tres problemas clásicos griegos que es la duplicación de cubos, este fue tratado por muchos años, aun así, hoy día los estudiantes tienen dificultad en el momento de graficar cónicas y también en la interpretación de dichas gráficas (Pérez, 2014). En el proceso de enseñanza, las cónicas son abordadas de manera superficial con herramientas básicas como los tableros y marcador, excluyendo la interacción directa entre la temática expuesta y el aprendiz (Aragón y Quintero, 2017).

En una síntesis sobre la base de las ideas expuestas, reconocemos una serie de dificultades en los estudiantes y docentes de matemáticas en formación de índole cognitivo, didáctico y epistemológico que se percibe de forma cotidiana en las instituciones educativas y universidades, afectando el desarrollo del proceso de enseñanza-aprendizaje considerablemente. En línea con lo anterior, esta investigación tiene como objetivo principal caracterizar los espacios geométricos personales de los profesores en formación en el contexto de problemas que involucran la articulación entre la geometría sintética y analítica. La investigación implicó un análisis a priori y uno a posteriori de cada uno de los docentes de matemáticas en formación sobre un problema geométrico.

\section{Método}

Esta investigación es de corte cualitativo (Martínez, 2006), se implementó la investigación clínica (Camargo, 2019), "en razón de que facilita la construcción de diseños investigativos del mundo empírico, permitiendo a los investigadores conectar sus supuestos teóricos con formas específicas de obtener información para analizarla" (p.2). Se implementó este método porque 
permite recolectar y analizar las formas de pensar de las personas que participan en entrevistas que incluyen preguntas del fenómeno en estudio.

Participaron cuatro estudiantes de último semestre matriculados al programa de Licenciatura en Matemáticas (futuros profesores de matemáticas) de una universidad pública del norte de Colombia. Se consideró la participación de los estudiantes de último semestre porque cursaron y aprobaron tres cursos de geometría (geometría I, geometría II y geometría analítica) y dos cursos de Tecnologías de la información y la comunicación (TIC) integrados a la educación matemática a lo largo del proceso de formación.

Se implementaron entrevistas semiestructuradas, videograbaciones y transcripciones, con el objetivo de recolectar y analizar las producciones escritas y orales de los participantes.

Las entrevistas evidencian los procesos mentales que en el pensamiento matemático incluye, pensamiento sobre tópicos matemáticos y procesos del pensamiento avanzados, como abstracción, justificación, visualización, estimación o razonamiento mediante hipótesis que deben operar sobre una red compleja de conceptos, unos avanzados y otros más elementales (Ramírez, Hernández y Prada, 2018). Todo esto en el plano de las ideas propias de un sujeto y sus significados, lo cual ayuda a exponer las ideas y procesos ocultos en el pensamiento del individuo que no pueden ser detectados con las demás técnicas (Clement, 2000 citado en Berrio, Fiallo y Acosta, 2013).

\section{Problema geométrico}

Se planteó la siguiente situación problema con el propósito involucrar al docente en la construcción del Espacio de Trabajo Geométrico personal (ETG personal), el cual es un entorno organizado por y para el geómetra. Diseñado en el contexto de la construcción del lugar geométrico parábola, un concepto que permite analizar la transición entre la geometría sintética y la geometría analítica.

Problema: Dada una recta $L$ cualquiera y un punto $F$ fuera de ella, construir puntos $P$ (20 puntos o más) tales que la distancia de $P$ a $F$ sea igual a la distancia de $P$ a la recta.

Después que el docente realice la tarea propuesta, se le solicita que encuentre una expresión analítica que describa el lugar geométrico. En el proceso de solución se implementa la entrevista semiestructurada, instrumento que permite recoger información y profundizar en las ideas plasmadas en las videograbaciones.

\section{Caracterización del Espacio De Trabajo Geométrico}

UnETG se refiere al entorno organizado por y para el geómetra (Kuzniak, 2004), el sujeto 
que se enfrenta a una tarea específica en geometría e involucra como parte de la actividad matemática la articulación de dos planos: el epistemológico y el cognitivo (ver figura 1). El plano epistemológico hace referencia a la utilización de un conjunto de objetos, posiblemente materializados en un espacio real y local, un conjunto de artefactos que serán las herramientas e instrumentos puestos al servicio del geómetra y finalmente, un repositorio teórico organizado de referencia, conformado por axiomas, definiciones y propiedades matemáticas. El plano cognitivo implica los procesos de visualización relacionado la representación del espacio y el soporte material, un proceso de construcción determinado por los instrumentos utilizados (reglas, compás, etc.) y las configuraciones geométricas mediadas por el proceso discursivo que produce argumentos y evidencia (Kuzniak, 2006).

Figura1.

Espacio de Trabajo Geométrico y sus génesis

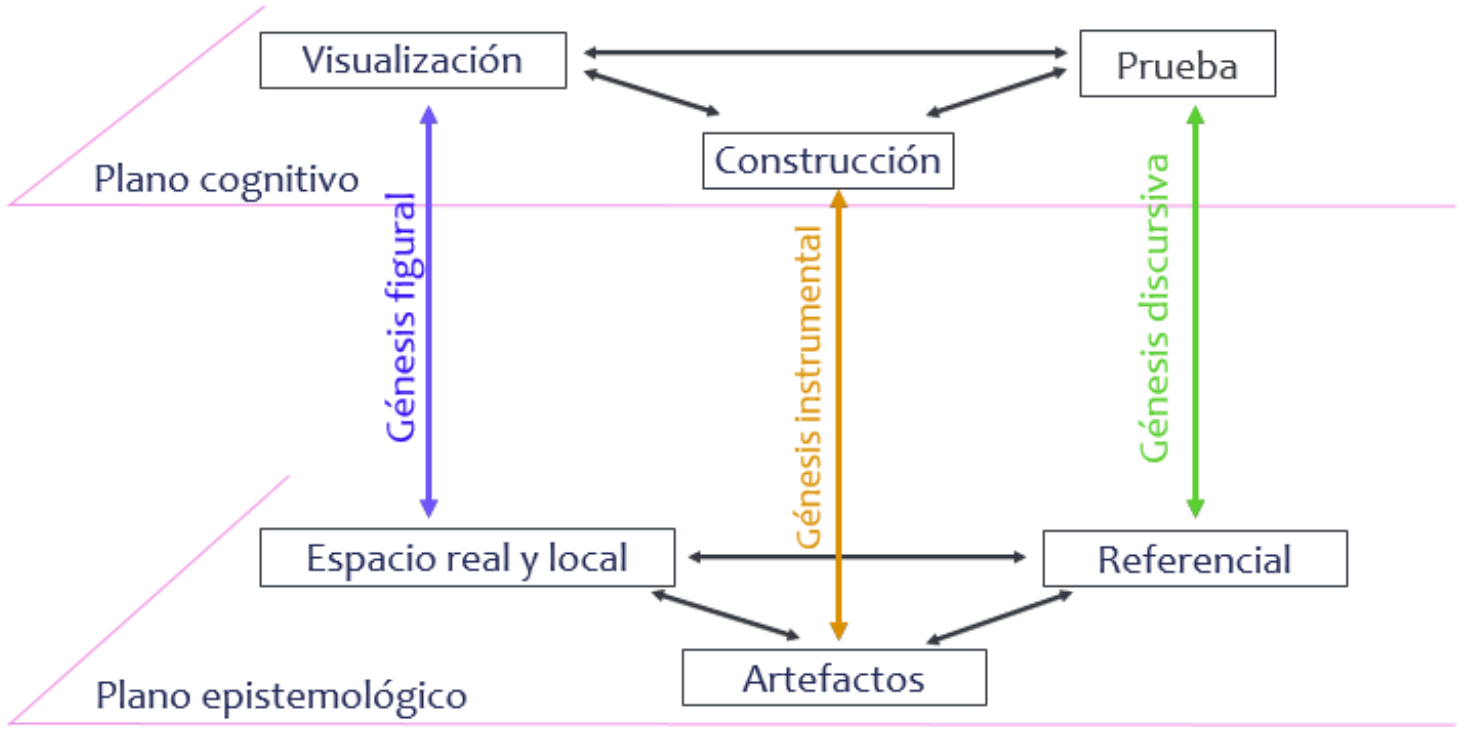

Fuente: Tomado de Kuzniak y Richard, 2010

El esquema que se presenta en la figura 1, modela el espacio de trabajo geométrico de un geómetra, constituido por dos planos que se articulan mediante tres génesis. Una génesis figural que describe el proceso semiótico entre el espacio real-local y la visualización, es decir, el significado que construye el individuo a través de la visualización de los signos y del objeto matemático lo cual le aporta soportes visuales privilegiados para describir el proceso y plantear estrategias de construcción. La génesis instrumental consiste en ilustrar las propiedades de los objetos estudiados mediante artefactos que permiten aumentar la dimensión procedimental y facilitar pruebas dinámicas que implican procesos cognitivos. En cuanto a la génesis discursiva, es aquella que asocia las formas geométricas a los conceptos geométricos mediante un discurso de explicación indispensable para argumentar basado en un sistema axiomático de referencia en donde el objeto geométrico no existe más que en y mediante una definición (Kuzniak, 2006; 2011; 2013). 
Investigaciones sobre ETG, señalan que el geómetra al resolver una situación problema de geometría, puede identificar tres tipos de trabajos geométricos: referencia, idóneo y personal. El primero, está definido de manera ideal en función de criterios geométricos utilizados por un experto epistémico. El segundo, concibe la reflexión sobre la reorganización didáctica de un objeto geométrico, definido en términos didácticos, lo emplea el profesor. El tercero, es el producto de la reflexión entre los conocimientos aprendidos y los puestos en prácticas, se tiene en cuenta los conocimientos y las capacidades cognitivas de sus utilizadores naturales que son el estudiante y el profesor (Kuzniak, 2006; 2011), este ETG es útil para identificar los saberes y dificultades de los estudiantes frente a una situación problema además de los procesos de razonamiento que desarrolla este para darle solución (Kuzniak y Rauscher, 2011).

Los datos recolectados se componen de transcripciones obtenidas a partir de las videograbaciones y la toma de notas auxiliares que se realizaron al implementar la situación problema a la muestra, estos relatos se convirtieron en material documental, produciéndose así escritos que permitieron analizar el fenómeno en estudio (Sánchez y Revuelta, 2005). Las producciones orales de los profesores fueron analizadas con base a los elementos que componen los espacios de trabajo geométricos, en particular, identificando en los extractos de las transcripciones expresiones referentes a las génesis figural, instrumental o discursiva que el docente activó para resolver el problema. Las producciones escritas, son el conjunto de evidencias que soportan la activación de las diferentes génesis en el espacio de trabajo personal de cada profesor. En línea con lo anterior, la interpretación de la información recolectada permitió registrar las experiencias y hechos relevantes para el desarrollo de la investigación y con ello realizar las respectivas descripciones de los componentes de las situaciones que se presentan en el trascurso de la solución del problema.

\section{Análisis de los datos}

En esta investigación el análisis de los datos implicó un análisis a priorì a uno a posteriori, el primer análisis permitió identificar situaciones que se pueden presentar en el proceso de ejecución del problema o relaciones a priori entre las variables y sus características, lo cual se confrontó con el resultado de la investigación (Orús, 1986). Los investigadores autores de este artículo resolvieron el problema propuesto en un momento inicial con el fin de identificar posibles respuestas, procedimientos, estrategias planteadas que permitieran deducir la construcción del lugar geométrico parábola que puede acontecer en la solución de la situación problema y reconocer aspectos a considerar para la implementación (ver Figura 2, 3y 4). 


\begin{tabular}{|c|c|}
\hline $\begin{array}{l}\text { 1. Es posible que algunos docentes en } \\
\text { formación ubiquen los puntos P utilizando la } \\
\text { opción del software "distancia o longitud" } \\
\text { entre dos objetos, en este caso los } \\
\text { investigadores solicitarán al docente en } \\
\text { formación que utilice } 15 \text { cifras decimales } \\
\text { para verificar su conjetura. Se espera que al } \\
\text { observar en la pantalla la diferencia entre las } \\
\text { medidas de los segmentos PI y PF, este } \\
\text { reformule su conjetura y busque otra forma } \\
\text { de darle solución al problema. }\end{array}$ & $\begin{array}{l}\text { Figura 2. Ubicación de los puntos } \mathrm{P} \text { con la } \\
\text { opción } \\
\text { "distancia }\end{array}$ \\
\hline $\begin{array}{l}\text { 2.Construir los puntos a partir de un objeto } \\
\text { geométrico (circunferencia, triángulo, } \\
\text { cuadrado) que verifique la equidistancia } \\
\text { entre los puntos } \mathrm{P} \text { y la recta L con los } \\
\text { puntos P y el punto F. Utilizando estos } \\
\text { objetos geométricos,el docente puede } \\
\text { ignorar la diferencia entre las definiciones de }\end{array}$ & $\begin{array}{l}\text { Figura 3. Construcción del lugar geométrico a } \\
\text { partir }\end{array}$ \\
\hline & Directriz \\
\hline $\begin{array}{l}\text { 3.La estrategia ideal para la construcción de } \\
\text { los puntos } \mathrm{P} \text {, involucra la construcción de la } \\
\text { mediatriz (recta de color naranja) del } \\
\text { segmento AF que comprende el punto fijo } \mathrm{F} \text { y } \\
\text { un punto cualquiera de la recta A por el cual se } \\
\text { traza una recta perpendicular (color amarillo). } \\
\text { Esto con el fin de determinar la intersección } \mathrm{P} \\
\text { de la recta perpendicular a } \mathrm{L} \text { y la mediatriz del } \\
\text { segmento AF (recta de color naranja). El punto } \\
\mathrm{P} \text { tiene la particularidad que equidista de la } \\
\text { recta L y del punto fijo } \mathrm{F} \text {. El lugar geométrico } \\
\text { parábola se genera cuando el punto A se } \\
\text { desplaza a lo largo de la recta L. }\end{array}$ & Figura 4. Construcción ideal del lugar \\
\hline
\end{tabular}

El proceso de ejecución del problema puede ser o no el esperado por el investigador, en consecuencia, se realiza un análisis a posteriori para contrastar la información (Berrío, 2016). 
Este tuvo como finalidad exponer e interpretar los hechos, las acciones, los razonamientos y las estrategias de solución propuestas y realizadas por el docente de matemáticas en formación frente al problema para luego realizar un contraste con el análisis a priori.

\section{Resultados}

Los resultados de la investigación refieren a una caracterización de los espacios de trabajo geométrico de los profesores de matemáticas en formación, en particular, presentamos los esquemas que especifican la articulación de los dos planos cognitivo-epistemológico y sus respectivos componentes, como son las génesis, las circulaciones y los polos que conforman los espacios de trabajo geométrico personales de cada docente.

\section{Docente 1}

En el contexto de la solución del problema de geometría, el docente inició activando la génesis instrumental, dado que pretendía identificar una figura geométrica (construcción) a través de medidas empíricas proporcionadas por el software (artefacto), aunado a esto, recurrió al espacio real y local que son las medidas de las distancias entre el foco $\mathrm{F}$ y un punto $\mathrm{P}$ cualquiera con las distancias de ese mismo punto $\mathrm{P}$ a la recta fija I, todo esto articulado con la visualización, en este recurre a la génesis figural.

[4] D1: Voy a utilizar el método de hilvanado para saber qué figura resulta.

[5] Inv: ¿En qué consiste el método de hilvanado?

[6] D1: Consiste en ubicar puntos en cierta medida aproximados... para crear un bosquejo de la figura que resulta de tales puntos.

Se evidencia que el docente emite una conjetura para justificar la equidistancia del punto $P$ al punto $\mathrm{F}$ y de $\mathrm{P}$ a la recta, para lo cual hace uso de las propiedades de la circunferencia mientras estimula la génesis instrumental en el proceso de construcción. Sin embargo, al validar su conjetura nota que el radio de la circunferencia no le garantiza la distancia del punto $\mathrm{P}$ a la recta, es decir, que acude a la génesis discursiva con el fin de darse cuenta que para garantizar la distancia de un punto a una recta necesita la distancia mínima y no la fórmula como tal.

[13] D1: Construye una circunferencia con centro en $\mathrm{P}$ y radio $\mathrm{F}$ que pasa por un punto de la recta $I$.

[14] D1: Así la distancia de P a F será igual a la distancia de P a la recta, por la propiedad del radio de la circunferencia.

[15] Inv: ¿Cómo se mide la distancia de un punto a una recta?

[16] D1: Con una fórmula, pero ahora no recuerdo...

[17] Inv: ¿Crees que necesitas la fórmula para garantizar la equidistancia? 
[18] D1: iNo, me equivoqué!

[19] D1: Necesito la distancia mínima del punto a la recta

El docente identifica un objeto geométrico que le permite garantizar la equidistancia, para ello realiza la construcción de dicho objeto y valida que las dos distancias sean iguales, en este episodio se evidencia la importancia de usar artefactos adecuados en un proceso de construcción, en este caso el software de geometría dinámica permite la construcción de la mediatriz y la perpendicular a la recta I cumplen ese papel de artefacto facilitador del proceso, que además de ser visualizado en la construcción, su definición y propiedades admiten una prueba crucial que concluye en la equidistancia de los dos segmentos.

[65] Inv: A partir de la construcción ¿Nos puedes dar una definición de ese lugar geométrico?

[66] D1: Todos los puntos que están sobre una parábola van a estar a la misma distancia o van a ser equidistantes del foco con la directriz, ¿sí?

[67] Inv: ¿Cómo puedes obtener una expresión analítica que represente ese lugar geométrico?

[68] D1: Con las fórmulas de distancias, de un punto a otro punto y de un punto a una recta, pero no recuerdo la de un punto a una recta.

En síntesis, el docente exploró con diferentes métodos para justificar la construcción de los puntos $\mathrm{P}$, uno de ellos fue la opción de medida del software y también la construcción de objetos geométricos, como se había previsto en el análisis a priori, además validó sus conjeturas a partir de las orientaciones dadas, transitó de la geometría sintética a la geometría analítica al articulular los elementos de los planos epistemológico y cognitivo (ver figura 5) , sin embargo, se le dificultó la construcción y prueba de una expresión algebraica de la parábola.

En la figura 5, se presenta la caraterización del ETG del profesor donde se evidencia que utilizó las genesis figural y las genesis instrumental para validar sus pasos de razonamiento, y logra activar la genesis discursiva al construir expresiones algebraicas sobre el lugar geométrico, pero se le dificulta deducir una expresión analítica concluyente para la cónica.

Figura 5.

Espacio de trabajo Geométrico personal del docente D1 de matemáticas en formación

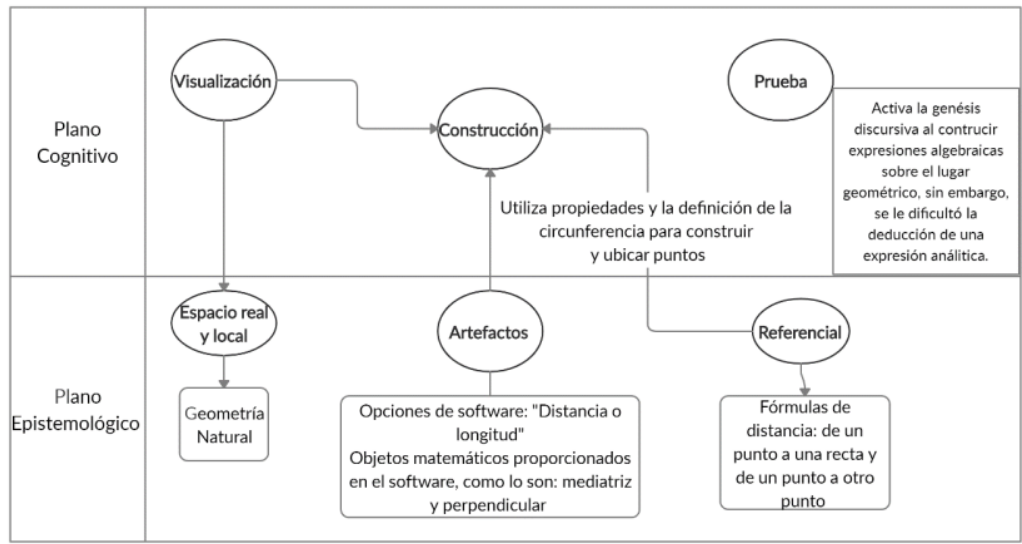




\section{Docente 2}

Con respecto al docente 2 en formación, validó los pasos de la construcción a través de la génesis figural dando mayor relevancia a las medidas de las distancias de forma aproximada, y no de manera numérica (Figura 6). El docente activó el polo de la visualización y empleó conceptos básicos de la geometría con dificultad para emplear esos conceptos en la transición de lo sintético a lo algebraico ya que omitió en el proceso de solución del problema la génesis discursiva, considerando el medio para validar el proceso de construcción.

Figura 6.

Espacio de trabajo Geométrico personal del docente D2 de matemáticas en formación

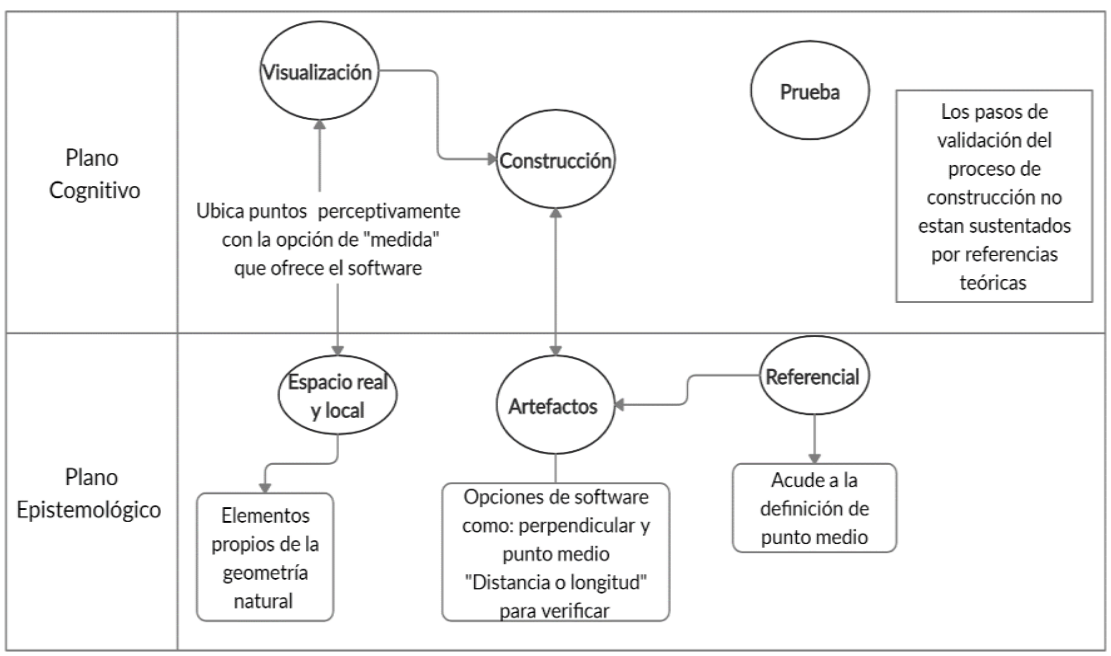

\section{Docente D3}

En el proceso de solución del problema del docente 3 en formación, desarrolló varias estrategias de solución para el problema con objetos geométricos, como se había previsto en el análisis a priori, pero tenía varias falencias en conceptos de geometría Euclidiana, lo cual le dificultó crear un espacio de trabajo personal apropiado para darle solución (Figura 7), sin embargo, con las orientaciones dadas en el desarrollo del proceso este logró realizar la construcción del lugar geométrico y lo identificó, pero al momento de hacer la transición de geometría sintética a la analítica el docente evitó recurrir a expresiones algebraicas que caracterizaban las distancias de un punto a otro y de un punto a una recta por lo cual tuvo que hacer uso de la web. Por otro lado, no tuvo en cuenta la orientación del plano cartesiano con respecto a la parábola, las coordenadas del foco de la parábola y tampoco la ecuación que representaba algebraicamente su directriz de esta manera no encontró la expresión analítica de la cónica. 
Figura 7.

Espacio de trabajo Geométrico personal del docente D3 de matemáticas en formación

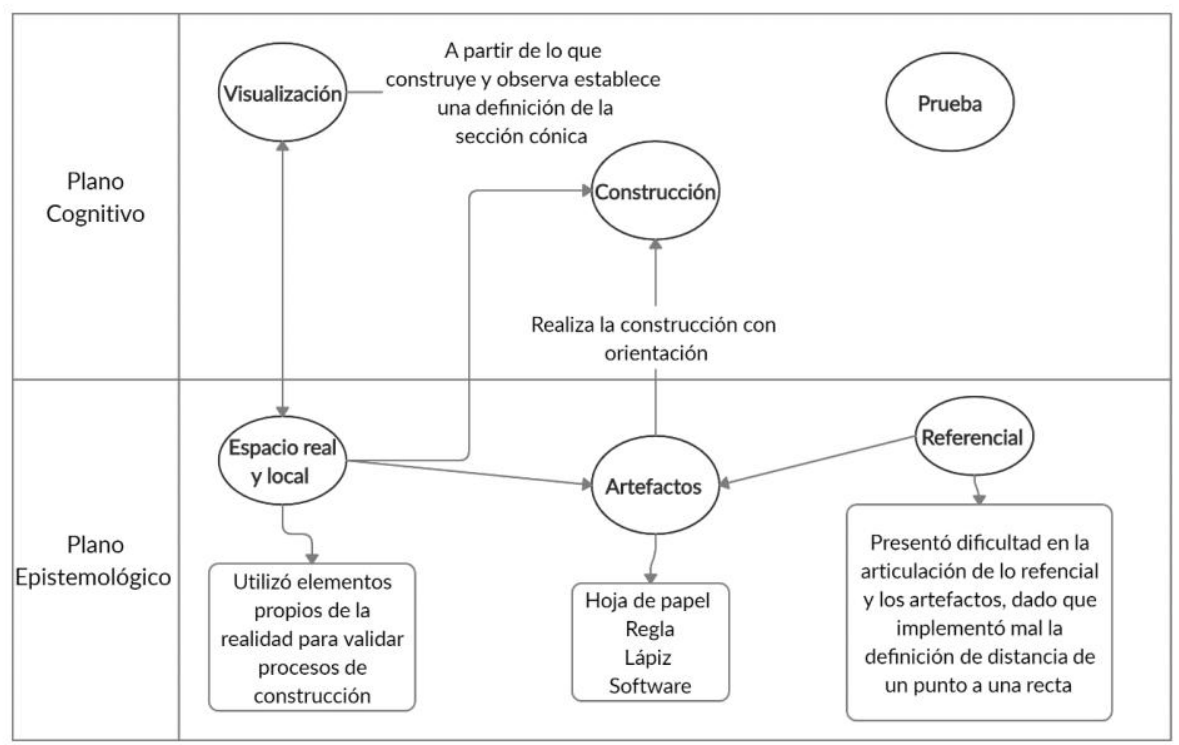

\section{Docente D4}

El docente 4 en formación evidenció conocimientos previos que a la hora de ponerlos en práctica le facilitó la construcción del lugar geométrico, dado a que el polo referencial mantuvo una circulación con el polo de prueba, esto se reflejó cuando el docente no recordó la propiedad de los puntos sobre la mediatriz, pero la dedujo a partir de pasos lógicos que lo llevaron a concluir a través de la congruencia de dos triángulos. Afirmó que el vértice $\mathrm{B}$ común equidista de los extremos del segmento FC por donde pasa la mediatriz, asimismo, el polo del espacio real y local mantuvo la circulación con la visualización en el momento de utilizar objetos geométricos y observarlos en el software, como también el polo artefactos en este caso en software y el polo construcción en la elaboración de la parábola, por consiguiente el docente articuló los dos planos del ETG tanto el cognitivo como el epistemológico con sus respectivas génesis, la figural, la instrumental y la discursiva (Figura 8). Reconocemos además que el docente logró la articulación de la geometría sintética con la geometría analítica al encontrar una expresión algebraica que representara dicho lugar geométrico. Sobre el paradigma geométrico privilegiado, el docente D4 se caracteriza con la geometría axiomática natural puesto que la manera de validar los conocimientos es por medio de razonamientos deductivos donde utiliza definiciones, propiedades y postulados. 
Figura 8.

Espacio de trabajo Geométrico personal del docente 04 de matemáticas en formación

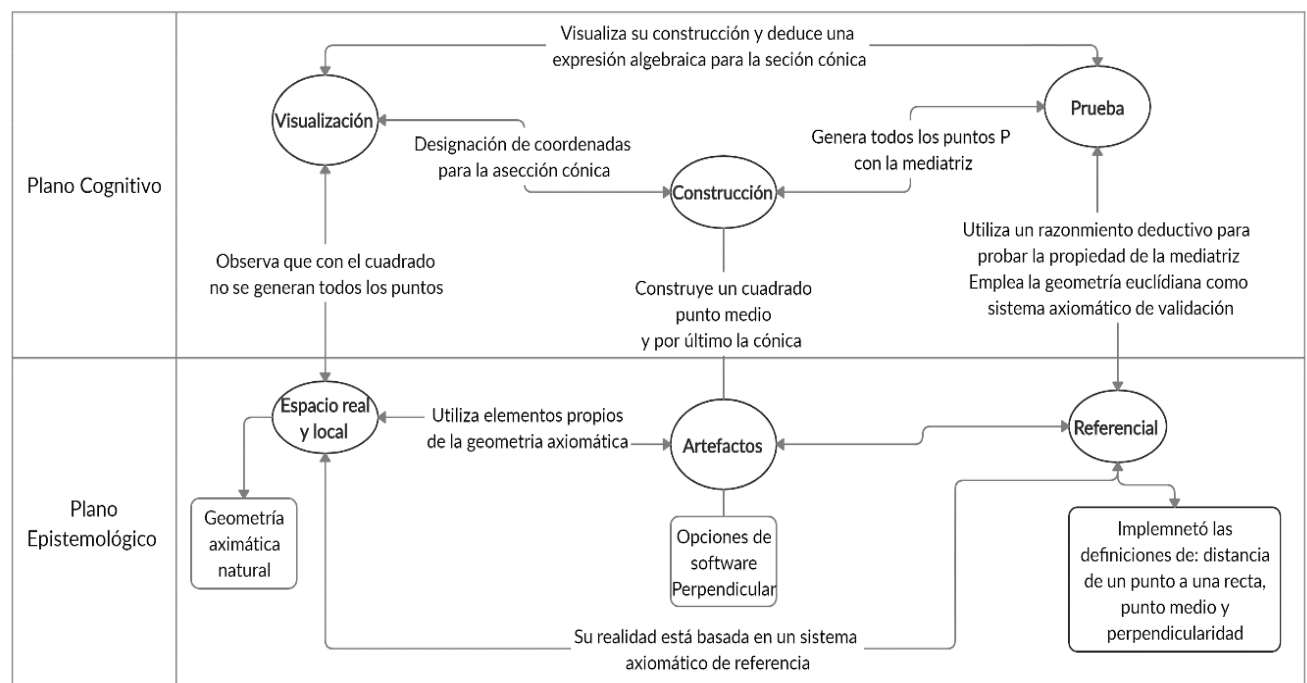

\section{Discusiones}

El centro de atención de esta investigación recae en caracterizar los espacios geométricos personales de los profesores de matemáticas en formación cuando resuelven problemas sobre geometría euclidiana y analítica. El conjunto de los resultados de la investigación son producto del análisis de las producciones orales y escritas de los profesores de matemáticas en formación que se caracterizan por ser un medio propicio para identificar las acciones realizadas por los docentes.

Una síntesis de los resultados nos lleva a establecer varios puntos esenciales a la luz de las investigaciones reportadas. Los espacios de trabajo matemático personales de los profesores permitieron reconocer elementos clave en la articulación entre la geometría euclidiana y analítica, los argumentos de los docentes son el medio por el cual se pudo reconstruir razonamientos, deducciones lógicas y con esto cada esquema que sintetizan los espacios de trabajo geométrico personales (Cervantes-Barraza, Ordoñez-Cuastumal y Morales, 2020).

Una interpretación de los resultados obtenidos permite reconocer que uno de los cuatro docentes realizó el tránsito entre elementos de la geometría euclidiana a la geometría analítica. Esto evidenció que los docentes que no transitaron entre los elementos de las geometrías presentan dificultades con el tratamiento de expresiones algebraicas y su desarrollo. En línea con lo anterior, estudios han documentado que tanto estudiantes universitarios y profesores presentan dificultades en traducir del lenguaje natural a lenguaje matemático (Ramírez Mora, Fregoso Vázquez, Calderón Mayorga, Cueva Gómez, Martínez Silva y Sánchez Díaz, 2008). Por su parte, Cervantes-Barraza, Valbuena y Paternina (2019) y Valbuena, Muñiz y Berrío (2020) 
señalan que se debe partir de la labor docente y contribuir en los procesos de enseñanza de la matemática teniendo en cuenta los resultados plasmados en la investigación.

Los medios que permitieron analizar y sintetizar los resultados de la investigación tienen que ver con los argumentos matemáticos que construyeron los docentes de matemáticas en formación. Se recomienda realizar investigaciones sobre los argumentos que permiten conectar el plano cognitivo con el epistemológico en el contexto de la solución de problemas. Se reconoce, además, que futuros docentes de matemáticas deben contribuir en el desarrollo de habilidades argumentativas lo que está relacionado con la comunicación matemática que implica reconocer el lenguaje propio de las matemáticas, usar las nociones y procesos matemáticos en la comunicación, reconocer sus significados, expresar, interpretar y evaluar ideas (Hernández, Prada y Gamboa, 2017). Este es un espacio para comunicar una problemática que puede ser investigada. Estudios recomiendan que los docentes implementen actividades que promuevan la argumentación en el salón de clases, esto conlleva a que mantengan una interacción entre pares y con esto, generen discusiones matemáticas en relación con los razonamientos de los demás estudiantes (Valbuena, Conde y Ortiz, 2018).

\section{Conclusiones}

El análisis de los esquemas que sintetizan los espacios de trabajo geométricos de los docentes es muestra de las falencias que tienen ante la solución de problemas que demandan la articulación entre conceptos básicos de la geometría euclidiana y la analítica. Estos recurrieron a estrategias de corte empírico para identificar un lugar geométrico, como el método del hilvanado y construcciones que carecían de sustento conceptual y axiomático. Sin embargo, reconocemos que el problema propuesto promovió la activación de los planos epistemológicos y cognitivos en cada docente, reacciones distintas fueron identificadas y sólo uno de los participantes abordó de manera eficaz entre la geometría euclidiana o sintética como vía exploratoria de las definiciones de geometría analítica, esto un medio que facilita la comprensión y apropiación de los conceptos de los lugares geométricos, en particular de la parábola.

Con base en los resultados obtenidos en esta investigación, se consideran algunas consideraciones importantes a la práctica docente. Resaltar que aplicar varias estrategias en la solución de un problema que involucra conceptos base y características invariantes del objeto matemático en estudio con el fin de fortalecer la articulación entre las geometrías ya que es posible justificar de manera abierta una misma situación. Los argumentos de los docentes son un medio pertinente para profundizar sobre aspectos cognitivos de los docentes, implementar métodos de análisis que faciliten la identificación y análisis de acciones que tiene que ver con los elementos de los espacios de trabajo geométrico.

En aras de establecer ideas para futuras investigaciones, recomendamos profundizar en la teoría de los ETG y la construcción de herramientas metodológicas propias de la teoría que faciliten la articulación de la geometría sintética con la geometría analítica. Además, es 
pertinente profundizar sobre los ETG idóneos y de referencias dado que conforman la totalidad de espacios descriptores de la actividad matemática ya sea desde el punto de vista del estudiante o del profesor.

\section{Referencias}

Álvarez, M. (2014). Relación Entre Geometría Sintética Y Analítica Y Tic's: Análisis Matemático Didáctico De Una Actividad [Trabajo de Grado de Especialización, Universidad Nacional de General Sarmiento, Buenos Aires].

Ancochea, B. (2011). Las funciones de las calculadoras simbólicas en la articulación entre la geometría sintética y la geometría analítica en secundaria. http://www.atd-tad.org/wpcontent/uploads/2012/05/Bernat-CITAD-III-2011.pdf

Aragón, J., y Quintero, C. (2017). Propuesta de enseñanza de las secciones cónicas usando diversas tecnologías para su desarrollo. [Trabajo de grado de Pregrado, Universidad Distrital Francisco José de Caldas, Bogotá].

Berrío, J. (2016). Estudio De La Construcción De Pasos De Razonamiento En El Proceso De Justificación Teórica En La Resolución De Problemas De Geometría [Trabajo de Grado de Maestría, Universidad Industrial de Santander, Bucaramanga].

Berrio, J.; Fiallo, J., y Acosta, M. (2013). Estudio de la construcción de pasos de razonamiento en el proceso de justificación teórica en la resolución de problemas de geometría. VII Congreso Iberoamericano de Educación Matemática, (1), 1611-1615.

Cabello, L. (2006). La enseñanza de la geometría aplicando los modelos de recreación y reflexión a través de la funcionalidad de materiales educativos. En Murillo, Manuel (Ed.), V FESTIVAL INTERNACIONAL DE MATEMÁTICA (pp. 1-12). Fundación CIENTEC..

Camargo, L. (2019). Estrategias cualitativas de investigación en Educación Matemática. XV Conferencia interamericana de Educación Matemática. https://conferencia.ciaemredumate.org/index.php/xvciaem/xv/paper/viewFile/1061/568

Cervantes-Barraza, J., Valbuena, S., y Paternina, Y. (2019). Argumentos de estudiantes de primaria en el contexto del álgebra temprana. Educación y Humanismo, 21(37), 120138. https://doi.org/10.17081/eduhum.21.37.3459

Cervantes-Barraza, J., Ordoñez-Cuastumal, J., y Morales, A. (2020). Argumentos de estudiantes universitarios en la solución de ecuaciones diferenciales ordinarias (EDO). Innovación educativa, 20(82), 83-103.

Del Río, C. (2018). La parábola como objeto matemático desde el enfoque ontosemiótico en el curso de matemáticas de grado decimo del instituto Mistrató Risaralda [Trabajo de Grado de Maestría, Universidad Tecnológica de Pereira]. 
Di Blasi, M., y Rodríguez, M. (2016). El fenómeno de la desarticulación entre los enfoques sintético y analítico en elipses: un estudio de caso. Revista electrónica de investigación en educación en ciencias, 11(2), 16-27.

Fernández, E. (2018). La geometría para la vida y su enseñanza. Aibi Revista de investigación, administración e ingeniería, 6(2), 34-63.

Gamboa, R., y Ballesteros, E. (2009). Algunas reflexiones sobre la didáctica de la geometría. Cuadernos de investigación y formación en matemáticas, 4(5), 113- 136.

Gascón, J. (2002). Geometría sintética en la ESO y analítica en el bachillerato. ¿Dos mundos completamente separados? Revista sobre la enseñanza y aprendizaje de las matemáticas, (2), 13-25.

Henríquez, C., y Montoya, E. (2015). Espacios de trabajo geométrico sintético y analítico de profesores y su práctica en el aula. Enseñanza de las Ciencias, (33.2), 51-70.

Hernández, C. (2012). Caracterización de la actividad demostrativa en estudiantes de educación superior. Revista Ecomatemático, 3(1), 37-43. https://revistas.ufps.edu.co/index.php/ecomatematico/article/view/118/131

Hernández-Suárez, C. A., Prada-Núñez, R., \& Gamboa-Suárez, A. A. (2017). Conocimiento y uso del lenguaje matemático en la formación inicial de docentes en matemáticas. Rev.investig.desarro.innov, 7(2), 287-299.

Itzcovich. (2005). Iniciación al estudio didáctico de la Geometría: de las construcciones a las demostraciones. Libros del Zorzal.

Kuzniak, A. (2004). Paradigmes et espaces de travail géométriques. (Note pour l'habilitation à diriger des recherches). Institute de Recherche sur l'Enseignement des Mathématiques Paris.

Kuzniak, A. (2006). Paradigmes et espaces de travail géométriques. Éléments d'un cadre théorique pour l'enseignement et la formation des enseignants en géométrie. Canadian Journal of Science and Mathematics Education, 6(2) 167-187.

Kuzniak, A. (2011). L'espace de Travail Mathématique et ses genèses. [El espacio de trabajo matemático y su génesis] Annales de didactique et de sciences cognitives, (16), 9-24.

Kuzniak, A. (2013) Understanding the nature of the geometric work through its development and its transformations. In Rezat, Hattermann \& Peter-Koop. (Eds.) Transformation - $A$ fundamental idea of Mathematics Education. (pp. 311-325). Springer.

Kuzniak, A., \& Rauscher J.C. (2011). How do teachers' approaches to geometric work relate to 
geometry students' learning difficulties? Educational Studies in Mathematics. 7イ(1), 129147.

Kuzniak, A., \& Richard, P. (2010). Espacios de trabajo matemático. Puntos de vista y perspectivas. Revista Latinoamericana de Investigación en Matemática Educativa, 13 (41), 1-11. https://doi.org/10.12802/relime.13.1741

Martínez, M. (2006). La investigación cualitativa (síntesis conceptual). Revista de Investigación en Psicología, 9(1), 123-146.

Montoya, E. (2014). El proceso de prueba en el espacio de trabajo geométrico: profesores en formación inicial. Enseñanza de las Ciencias, (2), 227-247.

Orús, P. (1986). L'Enseignement des Mèthodes de Classification. Proposition d'une ingénierie pour le cours moyen. I. R. E. M de Bordeaux, (Ed.) Collection Études en Didactique des Mathématiques.

Pérez, U. (2014). Estrategia didáctica para introducir las coordenadas polares y sus aplicaciones en la representación y análisis de la parábola y la elipse [Trabajo de Grado de Maestría, Universidad Nacional de Colombia].

Ramírez Mora, E., Fregoso Vázquez, R., Calderón Mayorga, C., Cueva Gómez, S., Martínez Silva, R., y Sánchez Díaz, S. (2008).Errores algebraicos más comunes en los estudiantes de primer ingreso de las carreras de Informática, Administración y Turismo del Centro Universitario de los Valles de la Universidad de Guadalajara. Educación y Humanismo, 10 (14), 23-39.

Ramírez, P., Hernández, C., \& Prada R. (2018). Elementos asociados al nivel de desarrollo del pensamiento lógico matemático en la formación inicial de docentes. Revista Espacios, 39(49), 11.

Ramírez, R. (2013). Las secciones cónicas en la escuela secundaria: un análisis matemático y didáctico. [Trabajo de Grado de Especialización, Universidad Nacional de General Sarmiento, Buenos Aires].

Sánchez, M., y Revuelta, F. (2005). El proceso de transcripción en el marco de la metodología de investigación cualitativa actual. Enseñanza, (23), 367-386.

Santaló, L. A. (1961). Geometría analítica y geometría sintética. Ciencia e investigación, 175), 145-154.

Valbuena, S., Conde, R., \& Ortiz, J. (2018). La Investigación en educación matemática y Práctica Pedagógica, perspectiva de licenciados en Matemáticas en formación. Educación y Humanismo, 20(34), 201- 215. https://doi.org/10.17081/eduhum.20.34.2593 
Valbuena, S., Muñiz, L., \& Berrío, J. (2020). El rol del docente en la argumentación matemática de estudiantes para la resolución de problemas. Revista Espacios, 41(9), 1-15. 